$$
g(z)=\frac{f(z)}{i e^{f(z) / i}},
$$

where $f(z)$ has been defined in $\S 3$. If the upper half plane is transformed conformally into the interior of the unit circle, $g(z)$ will be transformed into a function $l(z)=u(z)+i v(z)$, analytic in the interior of this circle. The function $l(z)$ takes on real values continuously on the circle except at one point where it takes on in the wide sense the value zero. In fact $f(z) / i$ is real along the real axis; it approaches zero uniformly as $z$ approaches the infinite point in such a way that $\phi$, the argument of $z$, satisfies the relation $0<\alpha<\phi<2 \pi-\alpha$ (see paragraph 3 ). Hence $g(z)$ approaches zero uniformly as $z$ approaches the infinite point subject to the above condition. It is further evident, since we know that $\lim f(z) / i=+\infty$ as $z$ becomes infinite along the positive real axis, that these boundary values are continuous, and therefore, a fortiori, summable.

If $l(z)$ has a normal Cauchy integral representation, then the absolute continuity of $\int_{\theta_{1}}^{\theta_{2}}|l(z)| d \theta$ is uniform for $r<1$. $^{*}$ Since $|v(r, \theta)| \leqq|l(r, \theta)|$, it follows that the absolute continuity of $\int_{\theta_{1}}^{\theta_{2}}|v(r, \theta)| d \theta$ is uniform for $r<1$. This is a contradiction since $v(r, \theta)$ (see Introduction) would vanish identically and this would imply that $l(z)$ is a constant, which it is not.

Houston, Texas

\title{
NOTE ON RELATIONS BETWEEN ELLIPTIC INTEGRALS AND SCHLÖMILCH SERIES
}

BY W. R. BENNETT

In a recent study of the problem $\dagger$ of calculating modulation products in the output of a rectifier when two sinusoidal waves of different frequencies are applied, a number of interesting and apparently novel mathematical relations were deduced as a byproduct of the analysis. Unless otherwise noted, $0 \leqq b \leqq a$, $a>0, a+b \leqq \pi$, and $m$ and $n$ may be any positive integers or zero. The following theorems are typical of the results obtained.

* Fichtenholz, Fundamenta Mathematicae, vol. 13 (1929), pp. 1-33.

$\dagger$ A paper discussing this problem and its solution has been prepared for publication elsewhere. 
I. If $m+n$ is odd, and $a+b<\pi$,

$$
\begin{aligned}
\sum_{r=1}^{\infty} \frac{J_{m}[(2 r-1) a] J_{n}[(2 r-1) b]}{2 r-1} & \Gamma\left(\frac{m+n}{2}\right) b^{n} \\
= & \frac{4 \Gamma(n+1) \Gamma\left(\frac{m-n+2}{2}\right) a^{n}}{F\left(\frac{m+n}{2}, \frac{n-m}{2} ; n+1 ; \frac{b^{2}}{a^{2}}\right) .}
\end{aligned}
$$

II. If $m+n$ is even, adding $\pi^{2} / 16$ to the right-hand side for $m=n$ $=0$, we have

$$
\begin{aligned}
\sum_{r=1}^{\infty} \frac{J_{m}[(2 r-1) a] J_{n}[(2 r-1) b]}{(2 r-1)^{2}} & \frac{\Gamma\left(\frac{m+n-1}{2}\right) b^{n}}{8 \Gamma(n+1) \Gamma\left(\frac{m-n+3}{2}\right) a^{n-1}} \\
= & \cdot F\left(\frac{m+n-1}{2}, \frac{n-m-1}{2} ; n+1 ; \frac{b^{2}}{a^{2}}\right)
\end{aligned}
$$

III. If $m+n$ is odd, adding $\pi^{2} a / 16$ to the right-hand side for $m=1, n=0$, and $\pi^{2} b / 16$ for $m=0, n=1$, we have

$$
\begin{aligned}
\sum_{r=1}^{\infty} \frac{J_{m}[(2 r-1) a] J_{n}[(2 r-1) b]}{(2 r-1)^{3}} & \Gamma\left(\frac{m+n-2}{2}\right) b^{n} \\
= & \frac{\Gamma \Gamma(n+1) \Gamma\left(\frac{m-n+4}{2}\right) a^{n-2}}{\cdot F\left(\frac{m+n-2}{2}, \frac{n-m-2}{2} ; n+1 ; \frac{b^{2}}{a^{2}}\right) .}
\end{aligned}
$$


It would be possible to generalize the above results to make the exponent in the denominator on the left-hand side any positive integer, but the tabulation of the exceptional cases, which increase in number as this exponent increases, would prove rather cumbersome.

In the series appearing above, the arguments of the Bessel functions are proportional to the rank of the term, and the series could, therefore, be technically regarded as Schlömilch series with one of the two Bessel functions in each term to be regarded as a part of the coefficient. Since the two Bessel functions play similar parts in the series, however, it seems preferable to regard these series as a more general type than the ordinary Schlömilch form which has only one Bessel function in each term; in fact the above series could be regarded as a degenerate case of a double Schlömilch series bearing somewhat the same relation to the ordinary Schlomilch series as the double Fourier series does to the single Fourier series. Apparently, series of the above nature have not as yet been studied to any extent in mathematical literature.

It will be noted that the first two terms in the argument of the hypergeometric functions are odd multiples of one-half, and that the third term is an integer. It follows that by the use of the difference equations for the hypergeometric function, and the relations

$$
K=\frac{\pi}{2} F\left(\frac{1}{2}, \frac{1}{2} ; 1 ; k^{2}\right), \quad E=\frac{\pi}{2} F\left(-\frac{1}{2}, \frac{1}{2} ; 1 ; k^{2}\right),
$$

the hypergeometric functions may be reduced to finite combinations of complete elliptic integrals with modulus $k=b / a$. A few typical results are the following:
(1) $\sum_{r=1}^{\infty} \frac{J_{1}[(2 r-1) a] J_{0}[(2 r-1) b]}{2 r-1}=\frac{E}{\pi}$;
(2) $\sum_{r=1}^{\infty} \frac{J_{0}[(2 r-1) a] J_{1}[(2 r-1) b]}{2 r-1}=\frac{E-\left(1-k^{2}\right) K}{k \pi}$;
(3) $\sum_{r=1}^{\infty} \frac{J_{0}[(2 r-1) a] J_{0}[(2 r-1) b]}{(2 r-1)^{2}}=\frac{\pi^{2}}{8}-\frac{a}{\pi}\left[2 E-\left(1-k^{2}\right) K\right]$; 
(4) $\sum_{r=1}^{\infty} \frac{J_{1}[(2 r-1) a] J_{1}[(2 r-1) b]}{(2 r-1)^{2}}=\frac{a}{3 \pi k}\left[\left(1+k^{2}\right) E\right.$

$$
\left.-\left(1-k^{2}\right) K\right]
$$

(5) $\sum_{r=1}^{\infty} \frac{J_{1}[(2 r-1) a] J_{0}[(2 r-1) b]}{(2 r-1)^{3}}=\frac{\pi^{2} a}{8}-\frac{a^{2}}{9 \pi}\left[\left(7+k^{2}\right) E\right.$

$$
\left.-4\left(1-k^{2}\right) K\right]
$$

(6) $\sum_{r=1}^{\infty} \frac{J_{0}[(2 r-1) a] J_{1}[(2 r-1) b]}{(2 r-1)^{3}}=\frac{\pi^{2} b}{8}-\frac{a^{2}}{9 \pi k}\left[\left(1+7 k^{2}\right) E\right.$

$$
\left.-\left(1+3 k^{2}\right)\left(1-k^{2}\right) K\right] \text {. }
$$

It may be remarked that when $b=0,(3)$ reduces to a Schlömilch series summation which was set as a problem* in the Mathematical Tripos of 1898.

The method by which the above results were derived consists of a comparison of various forms of the general coefficient in the double Fourier series expansion of the periodic function of two variables,

$$
f_{r}(x, y)=\left\{\begin{array}{rr}
-(P \cos x+Q \cos y)^{r}, & P \cos x+Q \cos y<0 \\
0 & , P \cos x+Q \cos y=0 \\
(P \cos x+Q \cos y)^{r}, & P \cos x+Q \cos y>0
\end{array}\right\} \text {, }
$$

where $r$ is zero or a positive integer. For convenience we take $P$ and $Q$ as positive, and $Q$ not greater than $P$. The function satisfies any one of several sets of sufficient conditions $\dagger$ for the expansion, which may be written:

$$
\begin{aligned}
f_{r}(x, y) & =\sum_{m=-\infty}^{\infty} \sum_{n=-\infty}^{\infty} C_{m n} e^{i(m x+n y)}, \\
C_{m n} & =\frac{1}{4 \pi^{2}} \int_{-\pi}^{\pi} \int_{-\pi}^{\pi} f_{r}(x, y) e^{-i(m x+n y)} d x d y .
\end{aligned}
$$

Although the application is of no importance in the mathematical argument, it may be of some interest to point out that when two sinusoidal waves of different frequencies are applied to a rectifier, the output may be expressed either as $(P \cos x$

* Watson, Theory of Bessel Functions, p. 634.

$\dagger$ Hobson, Theory of Functions of a Real Variable, vol. 2, Chap. VIII. 
$+Q \cos y) f_{0}(x, y)$ or $f_{1}(x, y)$ for a full wave linear rectifier, as $(1 / 2)\left[f_{1}(x, y)+P \cos x+Q \cos y\right]$ for a half wave linear rectifier, and as $(1 / 2)\left[f_{2}(x, y)+(P \cos x+Q \cos y)^{2}\right]$ for a halfwave square law rectifier, provided that we assign the special values to $x$ and $y, x=p t+\theta_{p}, y=q t+\theta_{q}, t$ being the time, $p, q$ the angular velocities, and $\theta_{p}, \theta_{q}$ the phase angles.

If we evaluate the integral (9) directly by determining the boundaries of the region where $P \cos x+Q \cos y$ is positive and integrating accordingly, we obtain expressions involving complete elliptic integrals. Instead of proceeding in this way, we may make use of the following Fourier series expansions,

$$
\begin{aligned}
& \frac{4}{\pi} \sum_{r=1}^{\infty} \frac{1}{2 r-1} \sin \frac{(2 r-1) \pi u}{c}=\left\{\begin{array}{rr}
-1,-c<u<0 \\
0, & u=0 \\
1, & 0<u<c
\end{array}\right\} \\
& -\frac{4 c}{\pi^{2}} \sum_{r=1}^{\infty} \frac{1}{(2 r-1)^{2}} \cos \frac{(2 r-1) \pi u}{c} \\
& =\left\{\begin{array}{r}
-u,-c \leqq u \leqq 0 \\
u, \quad 0 \leqq u \leqq c
\end{array}\right\} ; \\
& c u-\frac{8 c}{\pi^{3}} \sum_{r=1}^{\infty} \frac{1}{(2 r-1)^{3}} \sin \frac{(2 r-1) \pi u}{c} \\
& =\left\{\begin{array}{r}
-u^{2},-c \leqq u \leqq 0 \\
u^{2}, \quad 0 \leqq u \leqq c
\end{array}\right\} .
\end{aligned}
$$

The left-hand members of $(10)-(12)$ represent $f_{0}(x, y), f_{1}(x, y)$, $f_{2}(x, y)$, respectively, if $u=P \cos x+Q \cos y$ and $c>P+Q$.

Substituting the resulting expressions in the integrand of (9), and interchanging the order of summation and integration (which may easily be justified), we obtain generalized Schlömilch series forms for $C_{m n}$. Formulas (1)-(6) may now be established by direct comparison with the elliptic integral expressions obtained for $C_{m n}$ by the first method.

To prove the more general formulas I-III, consider the trigonometric integrals,

$$
\frac{2}{\pi} \int_{0}^{\infty} \frac{\sin u \lambda}{\lambda} d \lambda, \frac{2}{\pi} \int_{0}^{\infty} \frac{d}{d \lambda}(\cos u \lambda) \frac{d \lambda}{\lambda}, \frac{2}{\pi} \int_{0}^{\infty} \frac{d^{2}}{d \lambda^{2}}(\sin u \lambda) \frac{d \lambda}{\lambda} .
$$


It is clear that if we set $u=P \cos x+Q \cos y$, these integrals represent, respectively, $f_{0}(x, y), f_{1}(x, y), f_{2}(x, y)$. Inserting the resulting expressions in the integrand of (9), interchanging the order of the integrations so that the infinite integration is performed last,* and finally interchanging the order of differentiation and finite double integration, we get expressions for $C_{m n}$ in terms of the infinite integral of Weber and Schafheitlin which is in turn reducible to a hypergeometric function. $\dagger$ Formulas I-III are then obtained by direct comparison with the results obtained by the first method.

It appears that by substituting different forms for the function $f_{r}(x, y)$ a wide variety of additional results may be obtained. For example, if we replace $f_{r}(x, y)$ by the simple function $P \cos x+Q \cos y$, we have $C_{10}=C_{-1,0}=P / 2, C_{01}=C_{0,-1}=Q / 2$, and $C_{m n}=0$ for all other values of $m$ and $n$. But the Fourier series

$$
\frac{2 c}{\pi} \sum_{r=1}^{\infty} \frac{(-1)^{r+1}}{r} \sin \frac{r \pi u}{c}=u, \quad(-c<u<c),
$$

represents $P \cos x+Q \cos y$ if $u=P \cos x+Q \cos y$ and $c>P+Q$. Calculating $C_{m n}$ from the series and comparing, we find

$$
\sum_{r=1}^{\infty} \frac{(-1)^{r+1}}{r} J_{m}(r a) J_{n}(r b)=0,
$$

provided $m+n$ is odd and greater than one, and $a$ and $b$ satisfy the same restrictions as specified for formulas I-III. For $m=1$, $n=0$, and $m=0, n=1$, the right-hand member becomes $a / 4$ and $b / 4$ respectively. Known forms of Schlömilch series which represent null functions may be deduced $\ddagger$ by setting $b=0$.

Bell Telephone Laboratories

* Justification of the interchange is a bit delicate, but ample theorems are available. See, for example, Hobson, Theory of Functions of a Real Variable, vol. 2, p. 348.

$\uparrow$ Watson, Theory of Bessel Functions, p. 401.

‡ See Watson, Theory of Bessel Functions, pp. 634-636. 